This item was submitted to Loughborough's Research Repository by the author.

Items in Figshare are protected by copyright, with all rights reserved, unless otherwise indicated.

\title{
Test methods for hydrophobic coatings on solar cover glass
}

PLEASE CITE THE PUBLISHED VERSION

https://doi.org/10.1109/PVSC.2017.8366246

PUBLISHER

(c) IEEE

VERSION

AM (Accepted Manuscript)

PUBLISHER STATEMENT

This work is made available according to the conditions of the Creative Commons Attribution-NonCommercialNoDerivatives 4.0 International (CC BY-NC-ND 4.0) licence. Full details of this licence are available at: https://creativecommons.org/licenses/by-nc-nd/4.0/

\section{LICENCE}

CC BY-NC-ND 4.0

\section{REPOSITORY RECORD}

Isbilir, Kenan, Bianca Maniscalco, Ralph Gottschalg, and Michael Walls. 2019. "Test Methods for Hydrophobic Coatings on Solar Cover Glass". figshare. https://hdl.handle.net/2134/25850. 


\title{
Test Methods for Hydrophobic Coatings on Solar Cover Glass
}

\author{
Kenan Isbilir, Biancamaria Maniscalco, Ralph Gottschalg and John Michael Walls \\ CREST, Wolfson School of Mechanical, Electrical and Manufacturing Engineering, Loughborough \\ University, Loughborough, Leicestershire, LE11 3TU, UK
}

\begin{abstract}
The world market for solar energy continues to expand. However, to be competitive with traditional energy sources, photovoltaic (PV) modules must be capable of continuous and reliable high performance. Performance losses occur due to the soiling of the cover glass on modules. Soiling can be reduced by using hydrophobic coatings. These decrease surface energy and thus minimize adhesion to soiling. These coatings can help reduce maintenance and retain consistent electrical output. It is not yet clear, how hydrophobic coatings can be assessed and compared. In this paper, test methods to simulate the stresses that coatings experience in their life-time are assessed. These test methods help to predict the durability and useful lifetime of the coatings when applied to solar cover glass. Various test methods from different standards have been applied to hydrophobic coated glass surfaces and optimized to simulate real-outdoor conditions. A sand impact test and a water drop simulation test have been devised to study the effect of sand and rain on hydrophobic performance and durability.
\end{abstract}

Index Terms - hydrophobic coating performance, photovoltaic (PV) anti-soiling, coating durability tests.

\section{INTRODUCTION}

Solar Photovoltaic (PV) modules need to be highly efficient and reliable to compete with conventional energy sources. Currently they are produced with a manufacturer's warranty period of 25 years [1]. It is crucial to maintain the maximum performance throughout their life-time.

Soiling on PV cover glass is a significant problem that affects performance and requires costly maintenance. Dust and grime accumulation is a complex phenomenon and is influenced by diverse site-specific environmental and weather conditions. Accumulation of debris such as dust, sand, birddroppings and water-stains (salts) cause a reduction of the incident solar irradiance to the PV absorber and degrade the efficiency of the module [2]. The presence of soiling causes a reduction in transmitted light into the module. It also leads to inhomogeneous shading with an increased possibility of triggering hot spots [3]. It causes a reduction in the power output, thus increasing the cost of energy production. The solar industry schedules periodic cleaning of the PV arrays in solar farms to prevent the build-up of soiling on the module cover glass. However, the ongoing cost of maintenance is an additional financial burden for the operator.

The surface of the PV cover glass can be coated with a thin layer that acts as an anti-soiling coating. This layer can either be hydrophilic [4], that has high surface energy which attracts sufficient water to clean the surface or hydrophobic that has low surface energy which repels the water and washes the soiling away. These coatings reduce soiling and minimise the frequency of the cleaning task. The coating must be transparent otherwise it would cause optical losses.

The cleaning action of hydrophobic coatings stems from their high water contact angle. When water impinges on the hydrophobic surface, droplets of almost spherical shape form. These then roll away, carrying away the dust and dirt [5], [6]. Hydrophobic coatings have the potential to play an important role in reducing the level of soiling by presenting a low surface energy for reduced adhesion. They also make cleaning and maintenance easier for retaining performance [7].

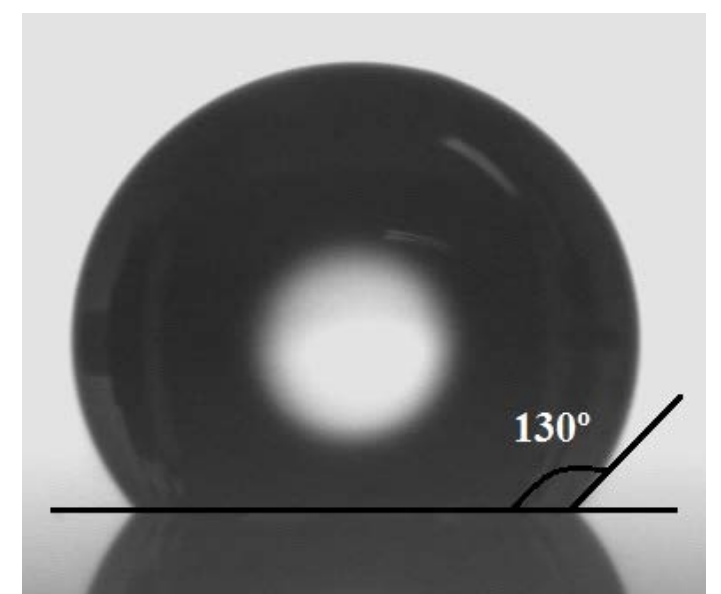

Figure 1-Measurement of the water contact angle on a glass surface with a hydrophobic coating

The life-time of hydrophobic coatings is crucial to sustain performance and ideally should match the life-time of the module. The coatings should resist the harshest outdoor conditions. The potential of hydrophobic coatings has already been recognized commercially and they are beginning to be deployed in the field. However, standards do not yet exist for performance and durability assessment. It is important that this is addressed to enable a fair and unbiased assessment of different anti-soiling coatings. In this work, methods to test and predict the durability and performance of hydrophobic coatings for PV cover glass are assessed. The results presented are obtained from hydrophobic coatings under development for application to solar cover glass. The formulation of the coatings was changed during these trials. However, the purpose of this study was to develop testing techniques for performance and durability. The results of tests on specific formulations will be published elsewhere. 


\section{EXPERIMENTAL}

Tests to assess the performance of hydrophobic coatings include optical transmission, water contact angle and roll-off angle. The durability tests include damp heat, ultraviolet (UV) exposure, thermal cycling, adhesion, abrasion resistance, solubility, sand impact and rain drop simulation, all of which simulate outdoor conditions or exposure to cleaning and maintenance. These tests have been performed on a variety of hydrophobic formulations. The test results reported are on hydrophobic coatings under development for commercial use by the PV industry. The focus has been on performance and not cost-effectiveness.

\section{A. Performance of Hydrophobic Coatings for PV Cover Glass}

The water contact angle (WCA) and the roll-off angle at a certain surface inclination determines the performance of hydrophobic coatings. In PV applications, the optical measurements also play an important role to avoid any absorption/reflection losses at the air-glass interface. Thus, the transmittance $(\mathrm{T})$ and reflectance $(\mathrm{R})$ measurements are considered important measures of coating performance. For simplicity, transmittance and reflectance measurements at 550 nm are used.

\section{B. Durability}

Durability is defined as the ability to withstand mechanical and environmental stresses. Hydrophobic coated cover glass surfaces were exposed to environmental stress tests such as damp heat, thermal cycling and UV exposure tests. These tests were carried out in accordance with IEC 61646:2008 to evaluate the scale and causes of degradation [8]. The surfaces were also exposed to various types of mechanical and chemical stress tests, including adhesion, resistance to abrasion, and water solubility.

The coated glass surface was visually inspected before and after each test. The optical measurements were obtained using a Varian Cary ${ }^{\circledR}$ UV 5000 spectrophotometer. The water contact angle was measured using a Dataphysics OCA 20 system after coating and at increasing time intervals during each of the accelerated durability tests. Optical and Scanning Electron Microscopy (SEM) images (Jeol® 7100F) were obtained to characterize the severity of the degradation. Rolloff angle was measured before and after each test with an inhouse developed measurement system, using between 20-40 $\mu \mathrm{l}$ water drops.

A sand impact test (SIT) was devised to evaluate durability to sand erosion. A new water drop test was developed to assess the impact of rain. In both of these devised tests, the samples were positioned on a platform at a $45^{\circ}$ angle, which is adjustable.

\section{RESULTS}

\section{A. Damp Heat Test}

The hydrophobic coatings were exposed to a damp heat test at $85^{\circ} \mathrm{C}$ and $85 \%$ relative humidity $(\mathrm{RH})$. The surface was exposed for a total of 1000 hours with intermediate optical and water contact angle measurements made at 100, 250 and 500 hours. The performance of the hydrophobic coating was severely reduced by the damp heat test after 1000 hours of exposure. Table I shows $\mathrm{T}$, R, roll-off angle and WCA measurements for initial, post 500 hours and final measurements until the surface lost the hydrophobic property which is defined when WCA is measured below $90^{\circ}$ [5]. The increase in roll-off angle shows that the damp heat exposure also increases the adhesion of the water drops. Despite degradation in hydrophobic performance, the optical performance showed a slight improvement associated with the effects of heat treatment on the coating.

TABLE I

DAMP HEAT TEST RESULTS

\begin{tabular}{|c|c|c|c|}
\hline & Initial & 500 hours & 1000 hours \\
\hline WCA ( $\left.{ }^{\circ}\right)$ & 106.3 & 90.7 & 83.1 \\
\hline Roll-off angle $\left(^{\circ}\right.$ ) & 73.0 & - & 78.2 \\
\hline R (\%) & 7.3 & 7.0 & 6.3 \\
\hline $\mathbf{T}(\%)$ & 92.9 & 92.8 & 93.1 \\
\hline
\end{tabular}

\section{B. Thermal Cycling Test}

The hydrophobic coatings were stressed using a thermal cycling test. One cycle comprises of 10 minutes of exposure to $85^{\circ} \mathrm{C}$ followed by 10 minutes at $-40^{\circ} \mathrm{C}$ with no humidity control according to the testing protocol IEC 61646:2008. The sample was exposed to a total of 200 cycles. The optical and hydrophobic measurement results in Table II shows that the coating was resilient to thermal cycling. The WCA measurements reveal a gradual degradation with increasing number of cycles with a reduction of $4.0 \%$ after 200 cycles. Transmittance and reflectance measurements show no significant change. However, an improvement in the roll-off angle is observed on exposure to thermal cycling.

TABLE II

THERMAL CyCLING TEST RESUltS

\begin{tabular}{|c|c|c|c|}
\hline & Initial & 100 cycles & 200 cycles \\
\hline WCA ( ${ }^{\circ}$ ) & 106.3 & 104.5 & 102.1 \\
\hline Roll-off angle ( ${ }^{\circ}$ ) & 72.9 & - & 50.1 \\
\hline R (\%) & 7.0 & 6.8 & 6.7 \\
\hline T (\%) & 93.1 & 92.9 & 92.9 \\
\hline
\end{tabular}




\section{UV Exposure}

The UV Exposure test was applied to hydrophobic coated surfaces for 500 hours with an intermediate check at 250 hours to determine its effect. The test was conducted with a minimum of $15 \mathrm{kWh} / \mathrm{m}^{2}$ of UV light with a $3 \%$ to $10 \%$ of the total energy in the UVB light range. The WCA measurement result shows that the UV exposure affects the hydrophobic performance of the coatings noticeably, but does not affect the optical performance significantly. The roll-off angle is increased slightly with UV exposure. This shows that the effect of UV exposure on roll-off angle and WCA can vary separately depending on the coating chemistry, as the two are not related [9]. The T, R, roll-off and WCA results are shown in Table III.

TABLE III

UV EXPOSURE TEST RESULTS

\begin{tabular}{|c|c|c|c|}
\hline & Initial & 250 hours & 500 hours \\
\hline WCA ( $\left.{ }^{\circ}\right)$ & 106.3 & 100.7 & 99.1 \\
\hline Roll-off angle ( ${ }^{\circ}$ ) & 72.5 & - & 79.4 \\
\hline R (\%) & 7.8 & 7.6 & 7.3 \\
\hline T (\%) & 92.7 & 92.3 & 92.4 \\
\hline
\end{tabular}

\section{Adhesion Test}

A tape adhesion test [10] was used to determine the adhesion of the coatings to the glass substrate. The surface was tested according to Mil-C-675C with minor changes. A 1inch long and 1inch wide adhesive tape was pressed firmly on the coated area for 10 seconds with an extra 1 inch to hold the tape. The tape is then removed rapidly within $1 \mathrm{~s}$. The results revealed no peeling or detachment of the coating. No change was observed to the hydrophobic and optical performance. The results shown in Table IV are consistent with good adhesion.

TABLE IV

ADHESION TEST RESULTS

\begin{tabular}{|c|c|c|}
\hline & Initial & Post exposure \\
\hline WCA ( $\left.{ }^{\circ}\right)$ & 106.3 & 105.9 \\
\hline Roll-off angle ( ${ }^{\circ}$ ) & 71.0 & 72.5 \\
\hline R (\%) & 7.9 & 8.0 \\
\hline T (\%) & 92.6 & 92.6 \\
\hline
\end{tabular}

\section{E. Abrasion Resistance Test}

The abrasion resistance test is important to assess the durability of hydrophobic coatings when exposed to maintenance or cleaning cycles. A test method similar to BS EN 1096-2 [11] was performed using a reciprocating abraser. A CS-10 grade abrader material [12] was used to simulate the degradation caused by particles. This provided a 'medium to hard' abrasion of the hydrophobic coating. Figure 2 shows an optical microscope image of an abraded area on the coating tested. The optical results are similar to the uncoated glass suggesting that in some cases most of the coating was removed during the test.

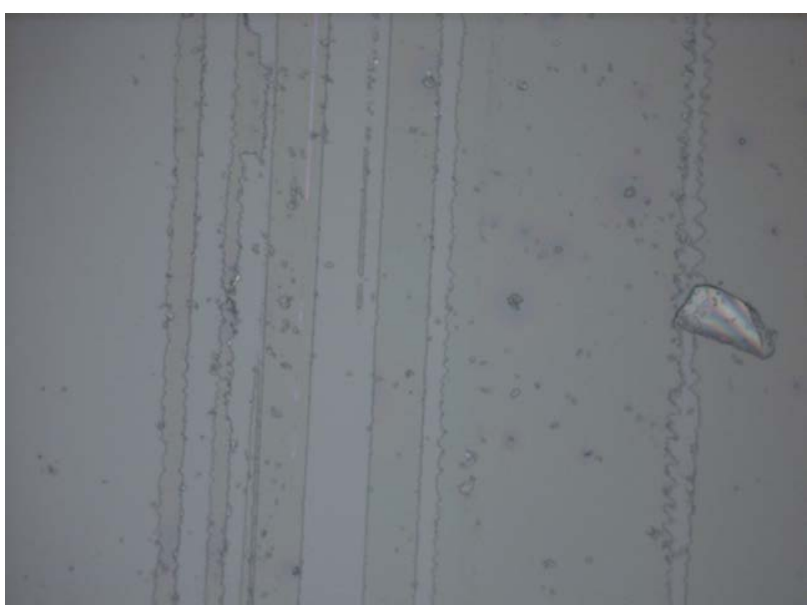

Figure 2-Optical Image of the coating after Abrasion Resistance Test

The initial and final results are shown in Table V. The WCA was reduced dramatically to below $90^{\circ}$. A significant increase in the roll-off angle of the coating could be caused by the increased roughness or hydrophobic coating removal in the abraded area.

Figure 3 shows the transmittance measurements over the 350 to $800 \mathrm{~nm}$ wavelength range for comparison with an uncoated glass sample. As seen on the graph, the transmittance of the coating (green) reduced to the level of the uncoated glass (amber) after the abrasion test.

TABLE V

ABRASION TEST RESULTS

\begin{tabular}{|c|c|c|c|}
\hline & Initial & 100 cycles & 200 cycles \\
\hline WCA ( $\left.{ }^{\circ}\right)$ & 104.8 & 76.5 & 70.2 \\
\hline Roll-off angle ( ${ }^{\circ}$ ) & 44.3 & 70.2 & 89.1 \\
\hline R (\%) & 7.2 & 8.3 & 8.3 \\
\hline T (\%) & 92.9 & 92.0 & 92.0 \\
\hline
\end{tabular}

\section{F. Solubility Test}

Hydrophobic coated samples were used to evaluate the applicability of a solubility test according to BS ISO 9211-4 [13]. There are 12 levels of severity for these tests. The severity can be increased gradually. The least severe exposure is immersion in DI water and the most severe exposure consists of immersion of the coating in boiling DI water for 2 minutes followed by immersion in DI water at room temperature for 1 minute. The coatings were exposed gradually to the most severe condition. Table VI shows the measurements after 10 cycles at the most severe exposure level.

Optical measurement results indicate that the coating had slight degradation in transmittance and reflectance. However a lower WCA was measured at the edges of the coated samples after tests at severity level 3. This is a total 96 hours of immersion in de-ionized (DI) water. Nevertheless, the coating preserved its hydrophobicity until testing at severity level 12. The post-exposure WCA results shown in Table VI are 
average values over the surface. The lowest was $99.6^{\circ}$ and highest was $118.5^{\circ}$ for the sample tested.

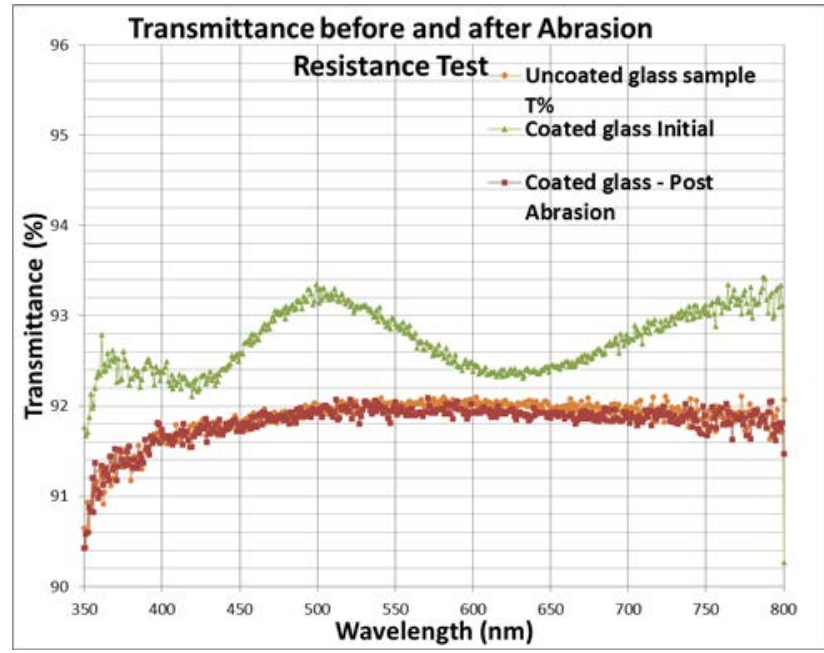

Figure 3-Measured transmittance before and after the Abrasion Resistance Test

TABLE VI

SOLUBILITY TEST RESULTS

\begin{tabular}{|c|c|c|}
\hline & Initial & Post exposure \\
\hline WCA ( $)$ & 118.6 & 104.4 \\
\hline Roll-off angle ( ${ }^{\circ}$ ) & 47.6 & 75.3 \\
\hline R (\%) & 4.7 & 5.3 \\
\hline T (\%) & 95.1 & 94.7 \\
\hline
\end{tabular}

\section{G. Sand Impact Test (SIT)}

Hydrophobic coated samples were exposed to $500 \mathrm{~g}$ of sand impacting the surface in 90s. The system was adopted from ASTM D968-15 [14]. The size of the sand particles used was $\sim 0.25 \mathrm{~mm}$. The results shown in Table VII correspond to the coating performance after sand exposure. The optical performance is measured approximately on the same point on each of the samples. The WCA results shown are the average of measured values over the coated area. The hydrophobic performance was not measured after the SIT without cleaning to avoid misleading results due to the retention of sand particles. The results after cleaning with pressurized air were compared with surfaces cleaned with IPA and DI water.

The SEM images in Figure 4-A and 4-B show the effect of abrasion on the surface of the coating. Some areas were only slightly damaged while on others the coating was completely removed. The optical and hydrophobic measurement results confirm the areas damaged by different degrees results in different levels of performance degradation. The lowest transmittance and reflectance measured over the coated area was $94.4 \%$ and $5.2 \%$, respectively. The lowest WCA measured post SIT after cleaning with air and with IPA/DI water was $62.5^{\circ}$ and $70.0^{\circ}$, respectively.
TABLE VII

SAND IMPACT TEST RESULTS

\begin{tabular}{|c|c|c|c|c|}
\hline & Initial & $\begin{array}{c}\text { Post SIT- } \\
\text { no } \\
\text { cleaning }\end{array}$ & $\begin{array}{c}\text { Post SIT- } \\
\text { cleaning } \\
\text { with air }\end{array}$ & $\begin{array}{c}\text { Post SIT- } \\
\text { cleaning with IPA } \\
\text { and DI water }\end{array}$ \\
\hline WCA ( ${ }^{\circ}$ ) & 119.5 & - & 84.6 & 92.8 \\
\hline Roll-off angle ( $\left.{ }^{\circ}\right)$ & 76.8 & - & - & $>90.0$ \\
\hline R (\%) & 2.8 & 4.4 & 3.4 & 4.2 \\
\hline T (\%) & 96.8 & 96.5 & 96.0 & 96.1 \\
\hline
\end{tabular}

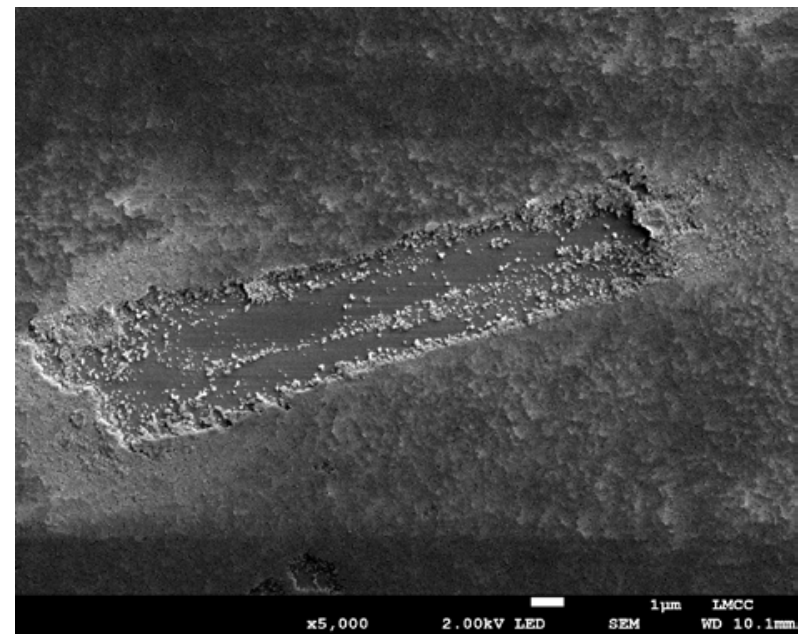

Figure 4-A - SEM image of a damaged area on the coating after Sand Impact Test

\section{H. Rain Drop Simulation Test (RDST)}

Deionized (DI), rain and tap water are used to simulate the effect of rain. The test was carried out for a continuous period of 1 minute to evaluate the coating performance in a controlled laboratory environment. In this way, outdoor factors such as temperature, dust etc. that may affect performance could be controlled. The diameter of the simulated drops was measured to be between 2 to $3 \mathrm{~mm}$ on a flat glass substrate. After exposure, the sample was left to dry under atmospheric conditions. The results show that the optical and hydrophobic performance was reduced mainly by water stains remaining on the surface after drying.

The results obtained using tap water are shown in Table VIII. The results indicate that the effect of water stains is highly detrimental to the coating performance. A slight increase in the WCA is possibly due to additional roughness caused by the exposure. However this additional roughness also caused an increase in roll-off angle. The water stains obstruct the movement of the water drop at roll-off. Figure -5 is the image of a coating surface after drying under atmospheric conditions. 


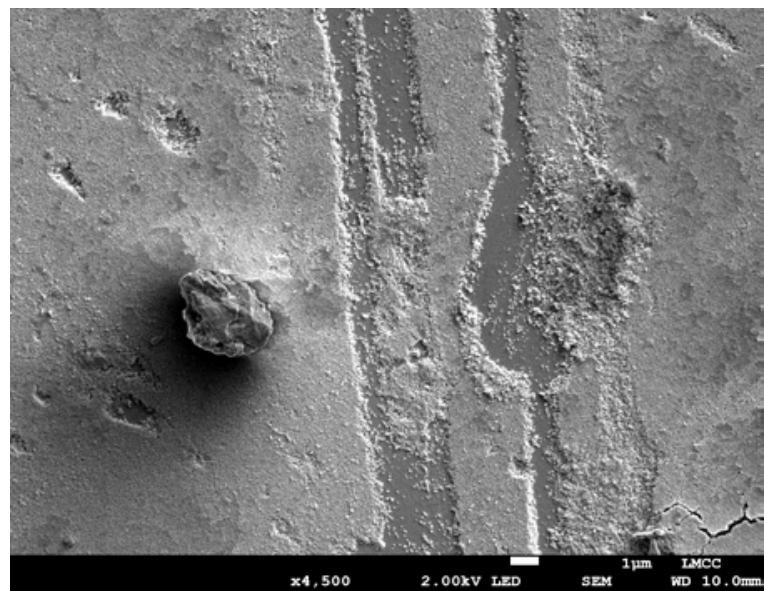

Figure 4-B - SEM image of partially damaged area after Sand Impact Test

TABLE VIII

RAIN DROP SiMULATION TEST (RDST) RESULTS

\begin{tabular}{|c|c|c|c|c|c|}
\hline & Initial & $\begin{array}{c}\text { Post } \\
\text { RDST- } \\
\text { highest }\end{array}$ & $\begin{array}{c}\text { Post } \\
\text { RDST- } \\
\text { lowest }\end{array}$ & $\begin{array}{c}\text { Post RDST- } \\
\text { after } \\
\text { cleaning }\end{array}$ & $\begin{array}{c}\text { Post RDST- } \\
\text { stain after } \\
\text { cleaning }\end{array}$ \\
\hline WCA ( ${ }^{\circ}$ ) & 118.9 & 128.0 & 52.0 & 125.0 & 90.0 \\
\hline Roll-off angle ( ${ }^{\circ}$ ) & 61.1 & - & $>90.0$ & 83.0 & $>90$ \\
\hline R (\%) & 1.3 & 1.1 & 5.4 & 1.5 & 1.4 \\
\hline T (\%) & 98.6 & 98.2 & 96.0 & 98.5 & 98.4 \\
\hline
\end{tabular}

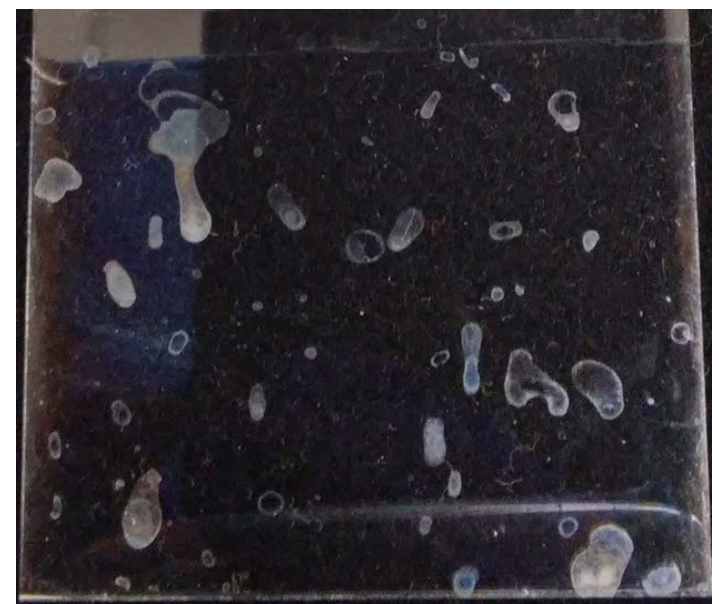

Figure 5-Surface of the coating post-RDST after drying at room temperature

\section{CONCLUSION}

Hydrophobic coatings on solar cover glass have the potential to minimize the accumulation of soiling and maintain module performance. The coatings are already becoming available commercially. Durability is a key concern. It will determine if the coatings will need to be re-applied and at what time interval. It is clear that durability will be dependent on the local environment. It is vital that the optical transmission of the coating is maintained. This paper considers the tests and standards that should be applied to hydrophobic coatings to ensure they are fit for purpose.

The environmental stress tests which are adopted from PV qualification standards are used to evaluate the degradation of hydrophobic coatings for PV modules. The test results show that the hydrophobic performance is degraded at different levels for different environmental stresses. Increase in optical performances was observed if heat is involved which acts as heat treatment of the coating surface.

Sand Impact Tests show how both optical and hydrophobic performance of coatings can suffer during the exposure. The coatings can be partially or completely removed. Optical measurements show that the point where the coating first suffered from sand impact has highest degradation. Variation between the WCA results over the coated surface was investigated using SEM. The images confirm the different effects of the sand impact at different locations.

The results from the rain drop simulation test emphasize that the roll-off angle should be below a 'critical value', so that the water drops clean the surface and reduce the risk of staining on the surface. Water stains are detrimental for the hydrophobic properties of coatings. Ideally, the roll-off angle should be below the installation angle of the PV modules.

\section{SUMMARY}

In this paper, the testing methods and performance requirements for hydrophobic coatings used on solar cover glass are compiled and assessed. The results of different environmental and mechanical stresses on hydrophobic coatings under development for commercial use by the PV industry are analysed. It is clear that the performance of the coatings may vary according to local environmental conditions. However, hydrophobic and optical performance should be consistent. The challenges for durability and performance of hydrophobic coatings in the field are highlighted.

\section{ACKNOWLEDGEMENT}

The authors are grateful to EPSRC for support of the SOLplus project funded through EP/N510014/1. The authors acknowledge use of facilities within the Loughborough Materials Characterisation Centre (LMCC).

\section{REFERENCES}

[1] A. Ndiaye, A. Charki, A. Kobi, C. M. F. Kebe, P. A. Ndiaye, and V. Sambou, "Degradations of silicon photovoltaic modules: A literature review," Sol. Energy, vol. 96, pp. 140-151, 2013.

[2] J. K. Kaldellis and P. Fragos, "Ash deposition impact on the energy performance of photovoltaic generators," J. Clean. Prod., vol. 19, no. 4, pp. 311-317, 2011. 
[3] H. Qasem, T. R. Betts, H. Müllejans, H. Albusairi, and R. Gottschalg, "Dust-induced shading on photovoltaic modules," Prog. Photovoltaics Res. Appl., vol. 22, no. 2, pp. 218-226, 2014.

[4] T. Lorenz, E. Klimm, and K. A. Weiss, "Soiling and anti-soiling coatings on surfaces of solar thermal systems - Featuring an economic feasibility analysis," Energy Procedia, vol. 48, pp. 749-756, 2014.

[5] H. Dodiuk, P. F. Rios, A. Dotan, and S. Kenig, "Hydrophobic and self-cleaning coatings," Polym. Adv. Technol., vol. 18, no. April, pp. 746-750, 2007.

[6] I. P. Parkin and R. G. Palgrave, "Self-cleaning coatings," J. Mater. Chem., vol. 15, no. 17, pp. 1689-1695, 2005.

[7] B. Brophy, Z. R. Abrams, P. Gonsalves, and K. Christy, "Field Performance and Persistence of Anti-Soiling Coatings on Photovoltaic Glass," in 31st European Photovoltaic Solar Energy Conference and Exhibition, 2015, pp. 2598-2602.
[8] IEC, "Thin-film terrestrial photovoltaic (PV) modules Design qualification and type approval,” 61646, 2008.

[9] N. K. Mandsberg and R. Taboryski, "The rose petal effect and the role of advancing water contact angles for drop confinement."

[10] Military Specification, "Coating of Glass Optical Elements (Anti-Reflection),” MIL-C-675C, 1986.

[11] BS EN, "Glass in building. Coated glass. Requirements and test methods for class A, B and S coatings," 1096-2, 2012.

[12] ASTM, "Standard Test Method for Abrasion Resistance of Organic Coatings by the Taber Abraser,” D4060-14, 2014.

[13] BS ISO, "Optics and photonics - Optical coatings, Part 4: Specific test methods," 9211-4, 2012.

[14] ASTM, "Standard Test Methods for Abrasion Resistance of Organic Coatings by Falling Abrasive,” D968-15, 2015. 